\title{
Percepções de tutores de instituições do Rio de Janeiro sobre as competências técnicas
}

André Tenório, Professor do Instituto Federal de Educação, Ciência e Tecnologia do Rio de Janeiro (IFRJ), tenorioifrj@gmail.com

Elaine dos Santos Lopes, Professora da rede municipal de Nova Iguaçu (RJ), nanilopes23@hotmail.com

Thaís Tenório, Pesquisadora da Universidade Aberta do Brasil (UAB/UFF), tenoriocalc@gmail.com

Resumo

As competências técnicas correspondem a aspectos relacionados à expertise do tutor nos conteúdos do curso em que atua e são úteis ao conduzir o processo de ensino-aprendizagem. Com o objetivo de identificar o papel dessas competências na atuação a distância, as percepções de dez tutores de cursos superiores do Rio de Janeiro foram colhidas por questionário. Para os pesquisados, a formação acadêmica fora essencial para desenvolver as competências técnicas, mas a experiência profissional permitira aperfeiçoá-las. Independentemente de o tutor atuar em cursos condizentes com sua área acadêmica, a formação pedagógica específica para educação a distância foi descrita como necessária. Os pesquisados consideraram as competências técnicas essenciais para a prática tutorial.

Palavras-chave: educação a distância, tutor, competências técnicas, didática.

\section{Perceptions of tutors at institutions in Rio de Janeiro on technical competencies}

\begin{abstract}
Technical competencies correspond to the tutor's expertise in course contents and play a significant role in conducting the teaching-learning process. Aiming to identify their importance to e-learning, perceptions of ten tutors at higher education institutions in Rio de Janeiro were collected through a questionnaire. The subjects considered that knowledge obtained in college was essential to develop their technical competencies, but professional experience helped improving them. Regardless of acting or not in courses akin to their own academic fields, specific pedagogical training for e-learning was deemed a ubiquitous necessity. The respondents considered the technical competencies essential in tutoring.
\end{abstract}

Keywords: e-learning, tutor, technical competencies, didactic.

\section{Introdução}

O crescimento da educação a distância $(\mathrm{EaD})$ é cada vez maior no Brasil (Sousa et al., 2011; Ramos, 2013). Um dos fatores para sua rápida expansão foi o emprego de tecnologias de informação e comunicação (TIC) (Mulbert et al., 2011; Ramos, 2013; Rosa, 2013; Tenório et al., 2014). Além disso, houve apoio governamental à EaD na política nacional, assim ela se tornou uma prioridade no plano de desenvolvimento da educação por favorecer a inclusão social e digital (Brasil, 2007).

$\mathrm{Na} \mathrm{EaD}$ contemporânea, a condução do processo de ensino-aprendizagem, marcadamente influenciado pelas TIC, é auxiliado pelo tutor - educador a distância que 
ajuda na construção do conhecimento pelo aluno ao atuar como conselheiro, mediador e orientador educacional. Ele estimula a interação, compartilhamento e colaboração entre alunos, além de estimular a participação ativa e posturas autônomas (Benetti et al., 2008; Teixeira et al., 2015; Tenório et al., 2015). Para exercer adequadamente suas funções, esse profissional deve possuir competências como o domínio do conteúdo técnico-científico da disciplina a ser tutorada (Belloni, 2009; Konrath et al., 2009).

Autores como Palloff e Pratt (2002), Tractenberg e Tractenberg (2007), Tecchio et al. (2008), Santana (2010), Bernardino (2011) e Ramos (2013) abordaram as competências técnicas em seus estudos, porém, nem todos definiram-nas similarmente. Alguns mencionaram apenas competências técnicas (Palloff; Pratt, 2002; Tecchio et al., 2008; Santana, 2010; Bernardino, 2011), outros distinguiram competências técnicas e tecnológicas (Tractenberg; Tractenberg, 2007; Ramos, 2013). Outros, como Villardi e Oliveira (2005) e Belloni (2009), denominaram competências técnicas de didáticas e as diferenciaram das tecnológicas. Assim, não existe uma definição consensual.

Neste estudo foram denominadas competências técnicas o domínio de conteúdos pertinentes à área disciplinar do tutor e a capacidade de transmiti-los. Por exemplo, um tutor de química precisa saber conteúdos de química o suficiente para conduzir o ensino-aprendizagem. Não obstante, a seguir são apresentados os pontos de vista de diferentes autores sobre as competências técnicas.

Palloff e Pratt (2002) classificaram as competências técnicas como a capacidade do tutor transmitir o domínio da tecnologia para seus alunos. No entanto, para o tutor conseguir transmitir conhecimentos tecnológicos, é fundamental que conheça bem a tecnologia (Machado; Machado, 2004). Santana (2010, p.8) reforçou o conceito acima, porém, utilizou o termo "cultura técnica", definido como o "domínio mínimo de técnicas ligadas ao audiovisual e à informática, indispensáveis em situações educativas cada vez mais midiatizadas”. Kemshal-Bell (2001) e Domingues, Gomes e Saragoça (2011) também citaram diversas competências tecnológicas como técnicas.

Para Tecchio et al. (2008), as competências técnicas seriam relacionadas a tudo que o profissional precisaria saber para bem desempenhar sua função. Essa definição, entretanto, é vaga, pois não especifica quais seriam tais saberes.

Bernardino (2011) descreveu as competências técnicas como referentes ao domínio dos recursos tecnológicos e a capacidade de socialização de saberes com os cursistas. Segundo alguns autores (Palloff; Pratt, 2002; Tecchio et al., 2008; Santana, 2010; Bernardino, 2011), a competência técnica tem a ver com a capacidade de utilizar a tecnologia como meio de trabalho, ou seja, o domínio das principais ferramentas que o tutor deveria utilizar para a comunicação com os alunos no decorrer do curso.

Contudo, outros discordam de tal classificação. De acordo com Tractenberg e Tractenberg (2007) e Ramos (2013), as competências técnicas e pedagógicas estariam relacionadas. Para eles, as competências técnicas seriam pertinentes ao domínio do conteúdo e dos materiais do curso. Villardi e Oliveira (2005) e Belloni (2009) consideraram as competências didáticas referentes à formação específica em uma área de conhecimento. Segundo Libâneo (2002), educadores afirmaram ser fundamental a tarefa de ensinar os alunos a pensar. Essa tarefa exige o desenvolvimento de estratégias para favorecer o ensino e a capacidade de reflexão. Nesse sentido, as competências técnicas também estariam relacionadas às pedagógicas, pois para ensinar seria preciso ter proficiência nos conteúdos ministrados para transmiti-los da melhor forma possível.

Na presente pesquisa, as competências técnicas, definidas de modo similar as de Tractenberg e Tractenberg (2007) e de Ramos (2013), englobariam: 
- Conhecer e compreender os conteúdos ministrados em um curso;

- Ser capaz de tirar dúvidas dos alunos sobre os conteúdos;

- Sugerir fontes para aprofundamento dos temas do curso;

- Promover reflexões críticas sobre os conteúdos;

- Mediar discussões sobre os conteúdos;

- Avaliar os trabalhos dos alunos.

$\mathrm{Na}$ EaD é imprescindível que o tutor tenha segurança sobre o conteúdo abordado no curso para orientar os alunos de forma clara e objetiva (Santos; Oliveira, 2013). As competências técnicas podem ser desenvolvidas através de saberes da formação acadêmica e da experiência profissional, definidos por Tardif (2002) e Oliveira e Sobrinho (2004) como:

- Saberes da formação profissional: transmitidos pelas instituições de ensino, pertencentes às ciências da educação e à ideologia pedagógica.

- Saberes experienciais: desenvolvidos na própria prática, no exercício das funções profissionais e que são incorporados à experiência individual e coletiva através do hábito e das habilidades (do "saber-fazer" e do "saber-ser").

Portanto, a formação acadêmica e complementar do tutor é de grande importância, pois irá refletir-se em sua atuação profissional, e, consequentemente, contribuir para a construção do conhecimento dos alunos.

Entender a importância atribuída por tutores ao conhecimento de conteúdos ministrados em seus cursos e à capacidade de transmiti-los pode ajudar a compreender o papel desse profissional na EaD. Foram investigadas as percepções de tutores do ensino superior de instituições brasileiras sobre a participação de competências técnicas na prática profissional. O objetivo da pesquisa foi conhecer, no âmbito da amostra, o valor das competências técnicas para a atuação tutorial, a aplicabilidade de tais competências e a necessidade de formação específica para o exercício profissional.

\section{Material e métodos}

As competências técnicas foram discutidas com base nas percepções de dez tutores, representadas pelas respostas obtidas por um questionário disponibilizado pela rede social Facebook.

\subsection{Coleta e análise dos dados}

A pesquisa seguiu uma abordagem qualitativa com dados colhidos por um questionário (Flick, 2005; Hill; Hill 2005; Yin, 2005). O instrumento de coleta de dados, elaborado pelos autores, foi organizado em duas partes com perguntas de respostas fechadas (objetivas) e semifechadas. Uma, com catorze perguntas, identificou o perfil do tutor e de sua prática profissional. Outra contou com treze perguntas sobre as competências técnicas.

O questionário foi disponibilizado por meio da rede social Facebook no segundo semestre de 2014. Os indivíduos que anuíram participar da pesquisa assinaram um termo de consentimento. Os dados foram quantificados a partir do padrão de respostas marcadas, tabulados e interpretados. Em questões com respostas semifechadas, a parte discursiva foi transcrita, sem necessidade de sistematizar e categorizar os dados. Não foi necessário usar técnicas de análise de conteúdo ou discurso. 


\subsection{Perfil dos participantes}

Dez tutores anuíram participar da pesquisa, sete mulheres e três homens de idades variadas, todos pós-graduados. Oito realizaram curso para atuar na $\mathrm{EaD} \mathrm{e}$ consideraram boa ou ótima a formação obtida.

Nove participantes tinham mais de 1 ano de experiência em tutoria. Os cursos tutorados eram de graduação ou especialização. Seis tutores atuavam na rede privada e quatro, na pública. Sete trabalhavam exclusivamente a distância, dois, de modo semipresencial e um, presencialmente (Tabela 1). A carga horária dedicada à tutoria (Tabela 1) era variada e pouco correlacionada ao número de disciplinas ou de alunos.

Os sistemas de gerenciamentos de ambientes virtuais de aprendizagem (AVA) usados eram o Moodle (4) ou o Epic (5).

Tabela 1. Dados sobre a atuação tutorial.

\begin{tabular}{c|c|c|c|c}
\hline Tutor & $\begin{array}{c}\text { Natureza de } \\
\text { instituição }\end{array}$ & $\begin{array}{c}\text { Nível do curso } \\
\text { tutorado }\end{array}$ & Tipo de tutoria & $\begin{array}{c}\text { Horas dedicadas à } \\
\text { tutoria }\end{array}$ \\
\hline A & pública & graduação & semipresencial & 5 a 10 horas \\
B & privada & graduação & $\begin{array}{c}\text { presencial } \\
\text { graduação }\end{array}$ & $\begin{array}{c}\text { semipresencial } \\
5 \text { a } 10 \text { horas }\end{array}$ \\
C & pública & graduação e & a distância & 16 a 20 horas \\
D & privada & especialização & a distância & 16 a 20 horas \\
E & privada & especialização & a distância & 5 a 10 horas \\
F & privada & especialização & a distância & 5 a 10 horas \\
G & privada & graduação & a distância & 11 a 15 horas \\
H & pública & graduação & a distância & 31 a 35 horas \\
I & privada & especialização & a distância & 16 a 20 horas \\
J & pública & graduação & &
\end{tabular}

\section{Resultados e discussão}

Quatro tutores consideraram as competências técnicas como a categoria de competências do tutor mais importante de todas, na frente de competências pedagógicas, socioafetivas, tecnológicas e gerenciais. Mas, no cômputo geral, as competências técnicas ficaram em segundo lugar de importância, superadas pelas pedagógicas.

Tabela 2. Atuação do tutor em sua área de conhecimento de graduação.

\begin{tabular}{cccccccccccc}
\hline Tutoria em disciplinas compatíveis & \multicolumn{1}{c}{ Tutor } \\
\cline { 2 - 11 } com a área de graduação & A & B & C & D & E & F & G & H & I & J & \\
\hline Sim & X & & X & X & X & & X & & & X & 6 \\
Não & & X & & & & X & & X & X & & 4 \\
\hline
\end{tabular}

Tabela 3. Atuação do tutor em sua área de pós-graduação.

\begin{tabular}{cccccccccccc}
\hline Tutoria em disciplinas compatíveis & & \multicolumn{1}{c}{ Tutor } & & & & \multirow{2}{*}{ Total } \\
\cline { 2 - 11 } com a área de pós-graduação & A & B & C & D & E & F & G & H & I & J & \\
\hline Sim & X & & X & X & X & X & X & & X & X & 8 \\
Não & & X & & & & & & X & & & 2 \\
\hline
\end{tabular}

Diversos autores, como Tardif (2002), Oliveira e Sobrinho (2004), Tractenberg e Tractenberg (2007) e Ramos (2013), destacaram a influência das formações acadêmica 
e complementar do tutor em sua atuação profissional. Para Belloni (2009), esse profissional deveria sempre atuar em sua área de conhecimento. A maioria dos tutores (8) julgou atuar em disciplinas compatíveis com as áreas de conhecimento de seu curso de graduação ou pós-graduação (Tabelas 2 e 3). Todavia, os participantes B e H declararam tutorar disciplinas incongruentes com as respectivas formações.

Tabela 4. Necessidade de formação pedagógica específica para atuar como tutor.

\begin{tabular}{cccccccccccc}
\hline Necessidade de formação pedagógica & \multicolumn{1}{c}{ Tutor } & & \multicolumn{1}{c}{ Total } \\
\cline { 2 - 10 } específica para atuar como tutor & A & B & C & D & E & F & G & H & I & J & \\
\hline Sim & X & & X & X & & & X & X & X & X & 7 \\
Não & & X & & & X & X & & & & & 3 \\
\hline
\end{tabular}

A maioria dos pesquisados (7) indicou ser necessário possuir formação pedagógica específica para atuar como tutor (Tabela 4). Belloni (2009) afirmou ser preciso o tutor possuir treinamento complementar para atuação profissional, sobretudo, na EaD. Só os participantes $\mathrm{B}$ e $\mathrm{G}$ não realizaram um curso para a atuação a distância, mas um deles (tutor a distância $\mathrm{G}$ ) ressaltou seu valor (Tabela 4).

Tabela 5. Tempo médio de resposta a dúvidas técnicas dos alunos.

\begin{tabular}{|c|c|c|c|c|c|c|c|c|c|c|c|}
\hline \multirow{2}{*}{$\begin{array}{l}\text { Tempo médio de resposta a } \\
\text { dúvidas técnicas de alunos }\end{array}$} & \multicolumn{10}{|c|}{ Tutor } & \multirow{2}{*}{ Tota } \\
\hline & $\mathrm{A}$ & $\mathrm{B}$ & $\mathrm{C}$ & $\mathrm{D}$ & $\mathrm{E}$ & $\mathrm{F}$ & $\mathrm{G}$ & $\mathrm{H}$ & I & $\mathrm{J}$ & \\
\hline 12 horas & & & & & & $\mathrm{X}$ & & & & & 1 \\
\hline 24 horas & $X$ & & $X$ & & $X$ & & & & $X$ & & 4 \\
\hline 36 horas & & & & & & & & $\mathrm{X}$ & & & 1 \\
\hline 48 horas & & & & $X$ & & & & & & $X$ & 2 \\
\hline 72 horas & & & & & & & $X$ & & & & 1 \\
\hline $\begin{array}{l}\text { Atuo em horários pré-fixados } \\
\text { pelo curso }\end{array}$ & & & & & & & & & & & $\underline{0}$ \\
\hline
\end{tabular}

O tempo médio de resposta a dúvidas técnicas de alunos era estimado em 35 horas (Tabela 5), menos de 2 dias. A dinâmica das atividades e da interação é largamente influenciada pelo tempo de resposta do tutor (Palloff; Pratt, 2004). Quanto maior o número de alunos, maior tendia a ser o tempo necessário para o tutor atender a todos. Os participantes D, G, H e J, atuantes em disciplinas com mais de 45 alunos, respondiam a dúvidas técnicas mais lentamente que os demais.

Tabela 6. Grau de conhecimentos técnicos requeridos no cotidiano como tutor.

\begin{tabular}{ccccccccccccc}
\hline Grau de conhecimentos técnicos & \multicolumn{1}{c}{ Tutor } & & & & \\
requeridos no cotidiano como tutor & A & B & C & D & E & F & G & H & I & J & Total \\
\hline Imprescindível & X & & & & & & & & & X & 2 \\
Elevado & & & X & & X & X & & X & X & & 5 \\
Razoável & & & & & & & X & & & & 1 \\
Baixo & & X & & X & & & & & & & 2 \\
Insignificante & & & & & & & & & & & \\
\hline
\end{tabular}

Para Tractenberg e Tractenberg (2007) e Ramos (2013), as competências técnicas envolviam o conhecimento dos conteúdos ministrados em um curso. Sete pesquisados reputavam como alto o grau de conhecimentos técnicos para bem 
desempenhar a tutoria (Tabela 6). Inclusive, muitos (8) recorriam comumente a saberes além dos curriculares para responder a dúvidas de alunos (Tabela 7). Os tutores B e D relataram precisar de pouco conhecimento técnico para a atuação profissional (Tabela 6) e raramente lançavam mão de conhecimentos além dos exigidos pelos cursos para resolver dúvidas (Tabela 7).

Tabela 7. Frequência com que o tutor recorreria a conhecimentos técnicos que extrapolam o exigido pelo curso ou disciplina para responder dúvidas de alunos.

\begin{tabular}{|c|c|c|c|c|c|c|c|c|c|c|c|}
\hline \multirow{2}{*}{$\begin{array}{l}\text { Frequência com que o tutor } \\
\text { recorreria a conhecimentos técnicos } \\
\text { que extrapolam o exigido pelo curso } \\
\text { para responder a dúvidas de alunos }\end{array}$} & \multicolumn{10}{|c|}{ Tutor } & \multirow[b]{2}{*}{ Total } \\
\hline & A & B & $\mathrm{C}$ & $\mathrm{D}$ & $\mathrm{E}$ & $\mathrm{F}$ & G & $\mathrm{H}$ & $\mathrm{I}$ & $\mathrm{J}$ & \\
\hline Sempre & & & & & $\mathrm{X}$ & & & & & & 1 \\
\hline Frequentemente & & & & & & $\mathrm{X}$ & $\mathrm{X}$ & & $\mathrm{X}$ & & 3 \\
\hline Âs vezes & $X$ & & $X$ & & & & & $\mathrm{X}$ & & $\mathrm{X}$ & 4 \\
\hline Raramente & & $\mathrm{X}$ & & $\mathrm{X}$ & & & & & & & 2 \\
\hline Nunca & & & & & & & & & & & $\underline{0}$ \\
\hline
\end{tabular}

De acordo com Tardif (2002) e Oliveira e Sobrinho (2004), as competências técnicas também poderiam ser desenvolvidas por meio da experiência profissional. Muitos (9) relataram precisar habitualmente de conhecimentos técnicos obtidos já durante a atuação para desempenhar adequadamente suas funções (Tabela 8). Apenas o pesquisado B, cuja tutoria era, basicamente, presencial, considerava raras as ocasiões em que precisava recorrer à sua experiência profissional.

Tabela 8. Grau com que o tutor recorreria a conhecimentos técnicos adquiridos somente após a formação acadêmica e durante a experiência profissional.

\begin{tabular}{|c|c|c|c|c|c|c|c|c|c|c|c|}
\hline \multirow{2}{*}{$\begin{array}{l}\text { Grau com que o tutor recorreria a } \\
\text { conhecimentos técnicos } \\
\text { adquiridos somente após sua } \\
\text { formacão acadêmica e iá durante } \\
\text { sua experiência profissional }\end{array}$} & \multicolumn{10}{|c|}{ Tutor } & \multirow[b]{2}{*}{ Total } \\
\hline & A & B & $\mathrm{C}$ & $\mathrm{D}$ & $\mathrm{E}$ & $\mathrm{F}$ & $\mathrm{G}$ & $\mathrm{H}$ & $\mathrm{I}$ & $\mathbf{J}$ & \\
\hline Sempre & & & & & $\mathrm{X}$ & & & & & & 1 \\
\hline Frequentemente & $X$ & & & & & & $X$ & $X$ & $X$ & & 4 \\
\hline Ầs vezes & & & $X$ & $X$ & & $X$ & & & & $X$ & 4 \\
\hline Raramente & & $X$ & & & & & & & & & 1 \\
\hline Nunca & & & & & & & & & & & $\underline{0}$ \\
\hline
\end{tabular}

Tabela 9. Frequência com que o tutor precisaria pesquisar antes de responder dúvidas dos alunos.

\begin{tabular}{ccccccccccccc}
\hline $\begin{array}{c}\text { Necessidade de pesquisar antes } \\
\text { de responder a dúvidas }\end{array}$ & A & B & C & D & E & F & G & H & I & J & Total \\
\hline Sempre & & & & & X & & & & & & & 1 \\
Frequentemente & & & X & & & & & & & X & 2 \\
Às vezes & & & & X & & X & & X & X & & 4 \\
Raramente & X & X & & & & & X & & & & 3 \\
Nunca & & & & & & & & & & & \\
\hline
\end{tabular}


Apesar dos saberes da formação acadêmica e da experiência profissional, grande parte (7) pesquisava antes de responder dúvidas de alunos (Tabela 9). Todavia, poucos se sentiam inseguros por falta de conhecimentos técnicos ao mediar debates (Tabela 10). Os pesquisados $\mathrm{B}$ e $\mathrm{H}$, que julgavam tutorar disciplinas incongruentes com as áreas de conhecimento de seus cursos de graduação ou pós-graduação (Tabelas 2 e 3), sentiam-se frequentemente inseguros durante a mediação de debates (Tabela 9). Segundo Santos e Oliveira (2013), o tutor deve dominar o conteúdo de seu curso para ser capaz de conduzir o processo de ensino-aprendizagem.

Tabela 10. Frequência com que o tutor se sentira inseguro ao mediar debates devido à falta de domínio de algum conteúdo em particular exigido no curso ou disciplina em que atua.

Frequência com que o tutor Tutor sentiria insegurança ao mediar os debates devido à falta de domínio de algum conteúdo

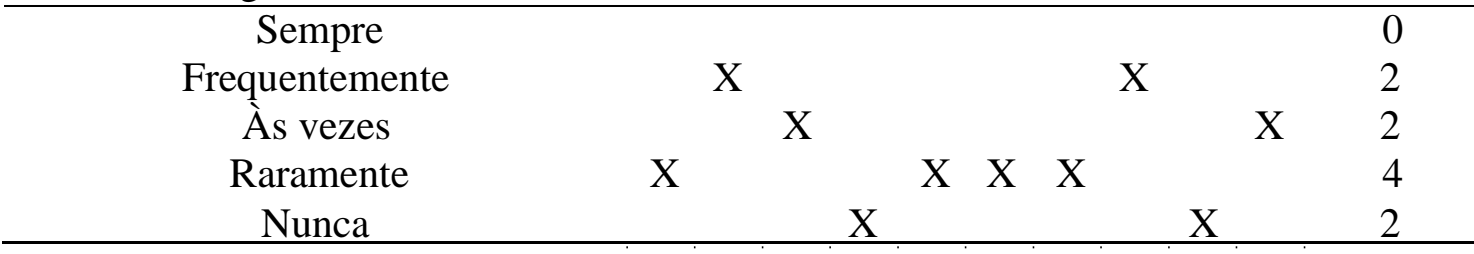

Grande parte considerava o nível técnico dos debates concordantes com seus conhecimentos (Tabela 11). Somente para o tutor E, o nível técnico dos debates sempre excedia seus saberes (Tabela 11), embora fosse mestre, treinado para atuar na EaD e experiente na tutoria. Contudo, ele raramente se sentia inseguro ao mediar os debates (Tabelas 10).

Tabela 11. Frequência em que o nível técnico dos debates mediados excederia os conhecimentos do tutor de um conteúdo particular.

\begin{tabular}{|c|c|c|c|c|c|c|c|c|c|c|c|}
\hline \multirow{2}{*}{$\begin{array}{l}\text { Frequência em que o nível técnico } \\
\text { dos debates mediados excederia os } \\
\text { conhecimentos do tutor }\end{array}$} & \multicolumn{10}{|c|}{ Tutor } & \multirow[b]{2}{*}{ Total } \\
\hline & A & $\mathrm{B}$ & $\mathrm{C}$ & $\mathrm{D}$ & $\mathrm{E}$ & $\mathrm{F}$ & G & $\mathrm{H}$ & $\mathrm{I}$ & $\mathrm{J}$ & \\
\hline Sempre & & & & & $\mathrm{X}$ & & & & & & 1 \\
\hline Frequentemente & & $X$ & & & & & & & & & 1 \\
\hline Às vezes & $X$ & & & & & & & & & $\mathrm{X}$ & 2 \\
\hline Raramente & & & $X$ & & & & & $X$ & $X$ & & 3 \\
\hline Nunca & & & & $\underline{X}$ & & $\underline{X}$ & $\underline{X}$ & & & & $\underline{3}$ \\
\hline
\end{tabular}

As principais dúvidas dos alunos, de acordo com as percepções dos tutores (Tabela 12), eram ocasionadas por falta de leitura das instruções passadas no AVA (6), dificuldades de interpretação de textos (5) e dificuldades de entendimento dos conteúdos das disciplinas (4). Para os pesquisados, nem todas as dificuldades dos alunos tinham relação com os conteúdos do curso. Muitas advinham da falta de atenção. 
Tabela 12. Percepções dos tutores sobre as principais dúvidas dos alunos.

\begin{tabular}{|c|c|c|c|c|c|c|c|c|c|c|c|}
\hline \multirow{2}{*}{$\begin{array}{c}\text { A maior parte das perguntas dos } \\
\text { alunos estaria relacionada a dúvidas }\end{array}$} & \multicolumn{10}{|c|}{ Tutor } & \multirow{2}{*}{ Total } \\
\hline & A & B & $\mathrm{C}$ & $\mathrm{D}$ & $\mathrm{E}$ & $\mathrm{F}$ & $\mathrm{G}$ & $\mathrm{H}$ & $\mathrm{I}$ & $\mathrm{J}$ & \\
\hline Técnicas sobre os conteúdos & & & & & & & & & & & \\
\hline $\begin{array}{l}\text { abordados especificamente na } \\
\text { disciplina que tutora }\end{array}$ & & $\mathrm{X}$ & $\mathrm{X}$ & & & & & $\mathrm{X}$ & $\mathrm{X}$ & & 4 \\
\hline $\begin{array}{l}\text { Técnicas remanescentes de disciplinas } \\
\text { anteriores }\end{array}$ & & $\mathrm{X}$ & & & $\mathrm{X}$ & & & & & & 2 \\
\hline $\begin{array}{l}\text { Ocasionadas por falta de estudo dos } \\
\text { materiais didáticos fornecidos }\end{array}$ & & & $\mathrm{X}$ & & & & & & & & 1 \\
\hline $\begin{array}{l}\text { Ocasionadas por falta de leitura atenta } \\
\text { das instruções fornecidas pelo tutor }\end{array}$ & & & & & & & & & & & 0 \\
\hline $\begin{array}{l}\text { Ocasionadas por falta de leitura atenta } \\
\text { das instruções passadas no AVA }\end{array}$ & & $\mathrm{X}$ & $\mathrm{X}$ & $X$ & $X$ & $\mathrm{X}$ & $\mathrm{X}$ & & & & 6 \\
\hline $\begin{array}{l}\text { Ocasionadas por dificuldades de } \\
\text { interpretação de textos em geral }\end{array}$ & $X$ & & $X$ & & $X$ & & & $X$ & & $\mathrm{X}$ & 5 \\
\hline $\begin{array}{l}\text { Ocasionadas por deficiências em } \\
\text { disciplinas básicas, como português e } \\
\text { matemática }\end{array}$ & & & $\mathrm{X}$ & & & & & $X$ & & & 2 \\
\hline Ocasionadas por desinteresse & & & & & & & & $\mathrm{X}$ & & & 1 \\
\hline
\end{tabular}

Tabela 13. Grau em que o tutor demonstraria conhecimento sobre literatura de sua disciplina.

\begin{tabular}{cccccccccccccc}
\hline Grau em que o tutor demonstraria & \multicolumn{1}{c}{$\begin{array}{c}\text { Tutor } \\
\text { conhecimento sobre a literatura }\end{array}$} & A & B & C & D & E & F & G & H & I & J & \\
\hline Elevado & & & X & X & & X & X & & X & & \\
Razoável & X & X & & & & X & & & X & & X & 5 \\
Baixo & & & & & & & & & & & 0 \\
Insignificante & & & & & & & & & & & 0 \\
\hline
\end{tabular}

As competências técnicas abarcariam a capacidade de o tutor promover reflexões críticas sobre os conteúdos e sugerir fontes para aprofundamento (Tractenberg; Tractenberg, 2007; Ramos, 2013). Tais habilidades demandam do tutor conhecimento sobre a literatura da disciplina tutorada. Metade dos pesquisados (5) julgou elevado o grau de conhecimento demonstrado aos alunos sobre a literatura de suas disciplinas. Os demais (5) consideraram-no razoável (Tabela 13). Sugerir leituras complementares era rotineiro na prática dos participantes (Tabela 14).

Tabela 14. Frequência com que o tutor indicaria leituras complementares aos alunos.

Frequência com que o tutor Tutor

\begin{tabular}{cccccccccccc} 
indicaria leituras complementares & A & B & C & D & E & F & G & H & I & J & Total \\
\hline Sempre & X & & X & & & & & & & & \\
Frequentemente & & & & & X & X & & & & X & 3 \\
Âs vezes & & & & X & & & X & X & & & 3 \\
Raramente & & X & & & & & & & X & & 2 \\
\hline
\end{tabular}

\section{Conclusões}

As competências técnicas do tutor podem ser definidas a partir de diferentes pontos de vista, como os propostos por Palloff e Pratt (2002), Tardif (2002), Oliveira e 
Sobrinho (2004), Tractenberg e Tractenberg (2007), Tecchio et al. (2008), Santana (2010), Bernardino (2011) e Ramos (2013). Nesta pesquisa, elas foram consideradas o domínio dos conteúdos didáticos pertinentes aos cursos tutorados, obtido durante formação acadêmica ou complementar ou da experiência profissional, conforme também estabelecido por Tardif (2002), Oliveira e Sobrinho (2004), Tractenberg e Tractenberg (2007) e Ramos (2013). As percepções de dez tutores de diferentes instituições de ensino superior do Rio de Janeiro sobre o emprego das competências técnicas na prática profissional foram identificadas por um questionário.

Para os respondentes, as competências técnicas estariam entre as mais importantes para o tutor, superadas apenas pelas pedagógicas. A maioria julgava atuar em disciplinas compatíveis com as áreas de conhecimento de seu curso de graduação ou pós-graduação. Entretanto, ainda assim, seria necessária formação pedagógica específica para tutoria a distância, de modo a melhorar o rendimento profissional. Conhecimentos técnicos obtidos durante a prática também contribuiriam para a atuação.

O grau de conhecimentos técnicos para bem desempenhar a tutoria foi reputado alto por muitos. Seria frequentemente necessário pesquisar ou recorrer a saberes além dos curriculares para responder às dúvidas. Conhecimento técnico seria indispensável à prática. Mas, na percepção dos tutores, as principais dúvidas dos alunos ocorreriam por motivos diversos, como falta de leitura das instruções passadas no AVA, dificuldades de interpretação de textos e dificuldades de compreensão dos conteúdos. O tempo médio de resposta a dúvidas de conteúdo foi estimado em 35 horas e, possivelmente, era influenciado pelo número de alunos assistidos. A insegurança por falta de conhecimentos técnicos ao mediar debates era incomum, talvez, porque o nível técnico dos debates era concordante com o saber dos pesquisados.

Como investigação complementar, poder-se-ia analisar e categorizar dúvidas passadas a tutores por correio de mensagens do AVA, e-mail e fórum de discussão. Então, a partir do levantamento, identificar a frequência relativa das dúvidas de caráter técnico e o tempo dispendido para satisfazê-las. E, assim, paralelamente por meio de entrevistas, tentar aferir subjetivamente o esforço dos tutores para respondê-las comparativamente às de outra natureza. Em um estudo futuro, seria interessante também pesquisar um maior número de tutores, com o intuito de tornar as evidências obtidas neste trabalho mais representativas em nível estadual ou nacional.

\section{Referências Bibliográficas}

BELLONI, M.L. Educação a distância. Campinas: Autores Associados, 2009.

BENETTI, K.C.; PACHECO, A.S.V ; MELO, P.A.; NAKAYAMA, M.K.; DALMAU, M.L.B.; SPANHOL, F.J.; TOSTA, H.T.; GIRARDI, D.M. Atuação docente na educação a distância: uma análise das competências requeridas. Revista Novas Tecnologias na Educação, Rio Grande do Sul, v. 6, n. 2, p. 1-11, 2008.

BERNARDINO, H.S. A tutoria na EaD: os papéis, as competências e a relevância do tutor. Revista Paidéi@, Santos, v. 2, n. 4, p. 1-7, 2011.

BRASIL. Referenciais de qualidade para educação superior a distância. Brasília: Ministério da Educação e Cultura, 2007.

DOMINGUES, M.J.C.S.; GOMES, G.; SARAGOÇA, V. Competências para a docência on-line: percepção de professores/tutores de pós-graduação no ensino a distância. In: ENCONTRO DE ADMINISTRAÇÃO DA INFORMAÇÃO, 3., 2011, Porto Alegre. Anais. Porto Alegre: SIGMESS, 2011. (CD-ROM). 
FLICK, U. Métodos qualitativos na investigação científica. Lisboa: Monitor, 2005. HILL, M.M.; HILL, A. Investigação por questionário. Lisboa: Edições Silabo, 2005. KEMSHAL-BELL, G. The on-line teacher. Final report prepared for the project steering committee of the VET Teachers and Online Learning, Department of Education and Training, Australia, 2001.

KONRATH, M.L.P.; TAROUCO, L.M.R; BEHAR, P.A. Competências: desafios para alunos, tutores e professores da EaD. Revista Novas Tecnologias na Educação, Rio Grande do Sul, v. 7, n. 1, p. 1-10, 2009.

LIBÂNEO, J.C. Adeus professor, adeus professora? Novas exigências educacionais e profissão docente. São Paulo: Cortez, 2002.

MACHADO, L.; MACHADO, E.C. O papel da tutoria em ambientes de EAD. In: CONGRESSO INTERNACIONAL DE EDUCAÇÃO A DISTÂNCIA, 11., 2004, Salvador. Anais. Salvador: ABED, 2004. (CD-ROM).

MULBERT, A.L.; GIRONDI, A.; PEREIRA, A.T.C.; NAKAYAMA, M.K. A interação em ambientes virtuais de aprendizagem: motivações e interesses dos alunos. Revista Novas Tecnologias na Educação, Rio Grande do Sul, v. 9, n. 1, p. 1-10, 2011.

OLIVEIRA, C.M.B.; SOBRINHO, J.A.C.M. Os saberes docentes na educação a distância: reflexões teóricas sobre a prática pedagógica do professor autor. In: ENCONTRO DE PESQUISA EM EDUCAÇÃO, 6., 2004, Teresina. Anais. Piauí: UFPI, 2004. (CD-ROM).

PALLOFF, R.M.; PRATT, K. Construindo comunidades de aprendizagem no ciberespaço: estratégias eficientes para a sala de aula on-line. Porto Alegre: Artmed, 2002.

RAMOS, M.S. Qualidade da tutoria e a formação do tutor: os efeitos desses aspectos em cursos a distância. In: CONGRESSO DE ENSINO SUPERIOR A DISTÂNCIA, 2013, Belém. Anais. Belém: UFPA, 2013. (CD-ROM).

ROSA, S.S. A influência das TDIC na (re)configuração de modelos de EaD tradicionais para educação online. Revista Novas Tecnologias na Educação, Rio Grande do Sul, v. 11, n. 3, p. 1-15, 2013.

SANTANA, R.R. Importância e competências requeridas do tutor no ensino de educação a distância. In: SIMPÓSIO REGIONAL DE EDUCAÇÃO, 1., 2010, Aracajú. Anais. Aracajú, 2010. (CD-ROM).

SANTOS, L.; OLIVEIRA, E.S.G. Tutoria em educação a distância: didática e competências do novo fazer pedagógico. Revista diálogo educacional, Curitiba, v. 13, n. 38, p. 203-223, 2013.

SOUSA, R.P.; MOITA, F.M.C.S.C.; CARVALHO, A.B.G. (Eds.). Tecnologias digitais na educação. Campina Grande: Universidade Estadual da Paraíba, 2011.

TARDIF, M. Saberes docentes e formação profissional. Petrópolis: Vozes, 2002.

TECCHIO, E.L.; MORETTO, S.M.; DALMAU, M.B.L.; NUNES, T.S.; MELO, P.A. Competências fundamentais ao tutor. In: CONGRESSO DA ASSOCIAÇÃO BRASILEIRA DE EDUCAÇÃO A DISTÂNCIA, 2008, Santos. Anais. Santos: ABED, 2008. (CD-ROM).

TEIXEIRA, N.; ALVES DE SALES, N.; TENÓRIO, T.; TENÓRIO, A. As competências socioafetivas aceitação e honradez segundo a percepção de tutores a distância. Revista Iberoamericana de educación a Distancia, Espanha, v. 18, n. 1, p. 129-149, 2015. 
TENÓRIO, A.; FERREIRA, R.S.L.; ALMEIDA, M.C.R.; ZUCON, L.H.; TENÓRIO, T. Ferramentas da educação a distância: a visão do tutor. EaD em foco, Rio de Janeiro, v. 4 , n. 1, p. 48-60, 2014.

TENÓRIO, A.; ROCHA, A.L.S.; TENÓRIO, T. Percepções de educadores sobre a contribuição de tecnologias de informação e comunicação para o ensino-aprendizagem a distância. Tecnologias na Educação, ano 7, n. 12, p. 1-11, 2015.

TRACTENBERG, L.; TRACTENBERG, R. Seis competências essenciais da docência online independente. In: CONGRESSO INTERNACIONAL DE EDUCAÇÃO A DISTÂNCIA, 22., 2007, Rio de Janeiro. Anais. Rio de Janeiro: ABED, 2007. (CDROM).

VILLARDI, R.; OLIVEIRA, E.G. Tecnologia na educação. Uma perspectiva sóciointeracionista. Rio de Janeiro: Dunya, 2005.

YIN, R.K. Estudos de caso: planejamento e métodos. Porto Alegre: Bookman, 2005. 